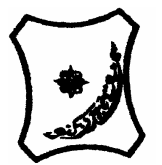

Bayero Journal of Pure and Applied Sciences, 6(1): 20 - 26

Received: April 2013

Accepted: May 2013

ISSN $2006-6996$

\title{
COMPARING HAEMOZOIN COUNT AND PARASITAEMIA IN THE PROGNOSIS OF SEVERE PLASMODIUM FALCIPARUM MALARIA IN CHILDREN AND NON-IMMUNE ADULTS IN KANO-NIGERIA
}

\author{
${ }^{1}$ Abubakar, A.G., ${ }^{2}$ Sarkinfada, F., ${ }^{3}$ Kwaru, A.H. and ${ }^{3}$ Ahmed, S.G. \\ ${ }^{1}$ Institute of Human Virology, Maina Court 252, Hebert Macaulay Way, Abuja Nigeria \\ ${ }^{2}$ Department of Medical Microbiology and Parasitology, Bayero University, Kano Nigeria \\ ${ }^{3}$ Department of Haematology, Aminu Kano Teaching Hospital, Kano Nigeria \\ $*$ Correspondence author
}

\begin{abstract}
This study compared the value of pigment containing leucocytes (neutrophils and monocytes) counts and the malaria parasite density in the prognosis of severe malaria on four hundred and twenty (420) patients with clinical evidence of severe malaria in Kano-Nigeria. Three clinical groups comprising patients with impaired consciousness, patients with cerebral malaria and those with severe anaemia were identified. Samples were analysed for Malaria parasitemia and pigment count Giemsa's thick and Leishman's thin film respectively. Patients with impaired consciousness $(n=217)$ recorded the highest malaria pigment count of $342.86( \pm 177.34)$ monocytes pigments/microliter. The highest parasite count of 234,962 ( \pm 264.5$)$ per microliter was recorded among the cerebral malaria group. Patients with severe malaria and anaemia had the least neutrophils pigment and parasite counts of 219.0 ( 140.96$) /$ microliter and 212,232( \pm 12.61$) /$ microliter respectively. A linear relationship between the malaria parasite count and the Intraneutrophilic malaria pigment count in severe malaria was demonstrated. Pigment count proved a higher prognostic value in severe malaria compared to Parasitaemia.
\end{abstract}

Keywords: Haemozoin, Plasmodium falciparum, Parasitaemia, Malaria

\section{INTRODUCTION}

Haemozoin or Malaria Pigment is produced by malaria parasites during intraerythrocytic development as the end product of haemoglobin digestion after Schizogony (Slater et al., 1991). The pigment consists of a polymer of haem group linked by iron-carboxylate bonds (Slater et al., 1991). After its formation; the insoluble malaria pigment is eventually phagocytosed by scavenger neutrophils and monocytes and is easily seen inside these cells by light microscopy (White et al., 1991; White et al., 1995; Amodu et al., 1998). Counting the number of pigments contained in peripheral blood phagocytes while examining the blood film for malaria parasites should give an indication of recent Schizogony in the deep vasculature and this could give a predictive value for malaria severity together with a prognostic value (White et al., 1995).

Parasite count has been used as a quantitative measure of infection and thus disease severity (Amodu et al., 1998). However, the clinical value of this prognostic relationship in the diagnosis of individual patients is limited by its poor predictive value. Some patients are able to walk with $50 \%$ of their red cells parasitized, while others die with Parasitaemia <0.1\% (MacPherson et al., 1985). The pathology of severe falciparum malaria is related to the sequestration of red blood cells containing mature forms of the parasite in the microvasculature of vital organ; whereas only immature, less pathogenic ring forms are seen in the peripheral blood film (MacPherson et al., 1985). Thus the severity of malaria is mainly due to the sequestrated parasite though not readily seen by the microscopists (White et al., 1991). As the relationship between the circulating and sequestered parasite burden is very variable, depending on the stage and synchronicity of infection, the relationship between Parasitaemia and disease severity is therefore a loose one (White et al., 1991; White et al., 1995).

In Plasmodium falciparum infection, the presence of pigment in the large mononuclear and/or polymorphonuclear calls for long and thorough search for the parasite. A mononuclear cell response is a very useful clue that anaemia is due to malaria (Topley, 1998).

A mild malaria pigment together with marked mononuclear response and often nucleated red cells provide the classical picture of severe anaemia due to malaria (Topley, 1998). Previous studies have shown malaria pigment proved a better indicator of prognosis than peripheral parasite count (White et al., 1991; White et al., 1995). In tropical areas, where unsupervised use of antimalarial drugs is common, patients with illnesses consistent clinically with severe malaria but with negative blood smears pose a management dilemma (White, 1992). Microscopy for intraleucocytic pigment is valuable in the differential diagnosis of severe febrile illnesses in malaria endemic areas where uncontrolled use to antimalarial drugs is widespread (White, 1992). In areas of high stable malaria transmission where adults with malaria are asymptomatic (premonition), severe infections are thought to occur only in young children (White et al., 1991). 
However, in a previous study, mortality was reported among $1.67 \%(1 / 60)$ of patients with severe malaria in Nigeria who had higher malaria pigment count in their leucocytes when compared with the survivors (Abubakar et al., 2002). Similarly, mortality among $40 / 300(13.3 \%)$ of adult patients with severe Malaria who had significantly higher proportions of malaria pigment - containing neutrophils on admission than the survivors were reported (Abubakar et al, 2002).

This work is aimed at comparing the malaria parasite density and the Intraleucocytic pigment count in the prognosis of severe $P$. falciparum malaria and disease severity among children and non-immune adults at Murtala Muhammad Specialist Hospital and Aminu Kano Teaching Hospital Kano Nigeria.

\section{MATERIALS AND METHODS Study Population:}

Four hundred and twenty (420) patients with severe malaria and / or impaired consciousness and malaria parasite ( $P$. falciparum) in their blood films were involved in the study. They comprised 70 adults and 350 children (among which 305 were males and 115 were females). The patients were recruited at the medical and pediatric wards of the Murtala Muhammad Specialist Hospital Kano, and Aminu Kano Teaching Hospital, Kano. Informed consent of the patients or relatives were obtained respectively before inclusion in the study.

\section{Inclusion and Exclusion Criteria:}

Patients with asexual form of $P$. falciparum in the blood film and any of the following features were eligible for the study:

i. Cerebral malaria with rousable or Unrousable coma.

ii. Severe (malaria) anaemia $(\mathrm{Hb}<5 \mathrm{~g} / \mathrm{dl})$

Patients who had received anti-malarials more than 12 hours before admission were excluded as well as those with no available Parasitaemia value after the first 2 treatment days. Evidence of morbidity or comorbidity with Cerebro Spinal Meningitis, hypoglycemia, and post ictal state were used to exclude patients from this study.

\section{Study Design}

The study is a cross-sectional study involving malaria cases with impaired consciousness and severe anemia as the sub group of patients with severe and complicated malaria reported within the study area and period. The subjects were sub-divided into three groups in accordance with Ejov et al. classification and grouped as follows:

Group 1: Patients with impaired consciousness: Glasgow score $>9 / 15$, with or without other severe malaria complications such as circulatory collapse and shock, spontaneous bleeding, renal failure or sickle cell anaemia.

Group 2: Patients with cerebral malaria: Unrousable coma, Glasgow score $<9 / 15$, with or without other severe malaria complications (such as pulmonary edema, circulatory collapse and shock, and fluid, electrolyte and acid-base disturbances).

Group 3: Patients with severe (normocytic normochromic) anaemia: Haemoglobin $<5 \mathrm{~g} / \mathrm{dl}$, in the presence of Parasitaemia, up to $10 \times 10^{3}$ per microliter and no other severe manifestations.

\section{Specimen Collection:}

Blood samples were collected into ethylene diamine tetra acetic acid (EDTA) containers on the first admission day of each patients or at least before the initiation of the antimalarial treatment. The blood was used for Packed cells Volume (PCV) estimation, haemoglobin estimation, total white cells count, film making for malaria parasite detection and parasite density estimation. Apart from film examination, all procedures were carried out within 2-3 hours of sample collection. The number of parasites counted per 100 leucocytes was multiplied by the total WBC of the patients to give a quantitative count per $\mu \mathrm{L}$ (White et al., 1991).

\section{Pigment Estimation:}

Haemozoin pigments were counted against 100 neutrophils and 30 monocytes and multiplied by the total WBC to express the pigments count per $\mu \mathrm{L}$ of blood.

Statistical Analysis: Statistical Analysis was done using Microsoft excel and Medcalc ${ }^{\mathrm{R}}$ statistical softwares to test for statistical significance of the difference in parasitaemia and pigment counts in the control and the three clinical group of patients.

Limitations: This study did not compare the three groups with mild or acute uncomplicated Malaria group (as additional control).

\section{RESULTS}

At the age of 1-10 years, it was observed that the ratio of male children with severe malaria is five times higher than that of the female (Table 1). We did not observe this relationship among the other age groups. A linear relationship was demonstrated among the three clinical groups between the neutrophil pigments count and Parasitaemia (per microliter each) this did not apply to the monocyte pigments count. There was no significant difference in Parasitaemia and leucocyte pigments count between the impaired consciousness and the cerebral malaria group $(\mathrm{P}=3.488)$ (Tables 2 and 3 ).

A comparison in the mean Parasitaemia between these three groups has also revealed a significant difference between both the cerebral malaria and impaired consciousness groups and the severe anaemia respectively $(P<0.001$ and $P<0.0001)$ (Table 5). Figures 1and2 show a Mann-Whitney's comparison in monocytic pigments count and neutrophils pigments counts among two clinical groups each. There was no significant difference in the monocytic pigments count between impaired consciousness and cerebral malaria groups using Mann-Whitney's $U$ test (Figure 1 ). However, these two groups have shown significant difference from the severe anaemia group using two tailed student's $t$ test $(P<0.0001)$ (Table 3$)$. The haemoglobin levels of all the three groups differ significantly from the control $(P<0.001, P<0.001$, $P<0.0001$ ) for groups 1 to 3 respectively (Table 4). A significant difference was observed in the Neutrophils pigments of impaired consciousness and severe anemia groups also using Mann-Whitney's $U$ test (Figure 2). 
Table 1: Age and Sex Distribution of the Patients with Severe Malaria in Kano Metropolis

\begin{tabular}{lll}
\hline Age group (years) & Male & Female \\
\hline $1-10$ & 251 & 52 \\
$11-20$ & 50 & 53 \\
$21-30$ & 4 & 5 \\
$31-40$ & 2 & 3 \\
$41-50$ & 0 & 0 \\
\hline
\end{tabular}

Table 2: Clinical, Parasitological and Hematological Features of Patients with Severe Malaria in Kano Metropolis

\begin{tabular}{|c|c|c|c|c|c|c|c|}
\hline Group & CF & $\mathbf{N}$ & $H(g / d l)$ & AP/ $/ \mu L$ & GC & $\begin{array}{l}\text { Mean } \\
\text { Neutrophil } \\
\text { Pigments/ } \mu \mathrm{L}\end{array}$ & $\begin{array}{l}\text { Mean } \\
\text { Monocytes } \\
\text { Pigments/ } \mu \mathrm{L}\end{array}$ \\
\hline $\mathbf{C}$ & $\begin{array}{l}\text { Apparently } \\
\text { Healthy }\end{array}$ & 60 & $13.0(1.5)$ & 0 & 0 & 0 & 0 \\
\hline 1 & $\begin{array}{l}\text { Impaired } \\
\text { Consciousness }\end{array}$ & 77 & $* 10.2(0.8)$ & $\begin{array}{l}233,861 \\
(342.3)\end{array}$ & $<9 / 15$ & $291.22(12.61)$ & $\begin{array}{l}342.86 \\
(121.26)\end{array}$ \\
\hline 2 & Cerebral Malaria & 126 & *8.9 (0.7) & $\begin{array}{l}234,962 \\
(264.5)\end{array}$ & $9 / 15$ & $302.10(177.34)$ & $326.0(120.33)$ \\
\hline 3 & Severe Anaemia & 217 & *6.2 (0.2) & $\begin{array}{l}* 212,232 \\
(249.4)\end{array}$ & 0 & *219.0 (140.96) & *269.0 (72.70) \\
\hline
\end{tabular}

Key: $\mathrm{C}=$ Control, $\mathrm{N}=$ Number, $\mathrm{CL}=$ Clinical features, $\mathrm{AP}=$ Admission Parasitaemia, $\mathrm{H}=$ Haemoglobin, $\mathrm{GC}=$ Glasgow Coma

Standard Deviation in Parenthesis, *Statistically significant difference (t-test)

Table 3: Students t-test for Significance of means of Leucocytic Malaria Pigment Count Among the Three Clinical Groups with Severe Malaria in Kano Metropolis

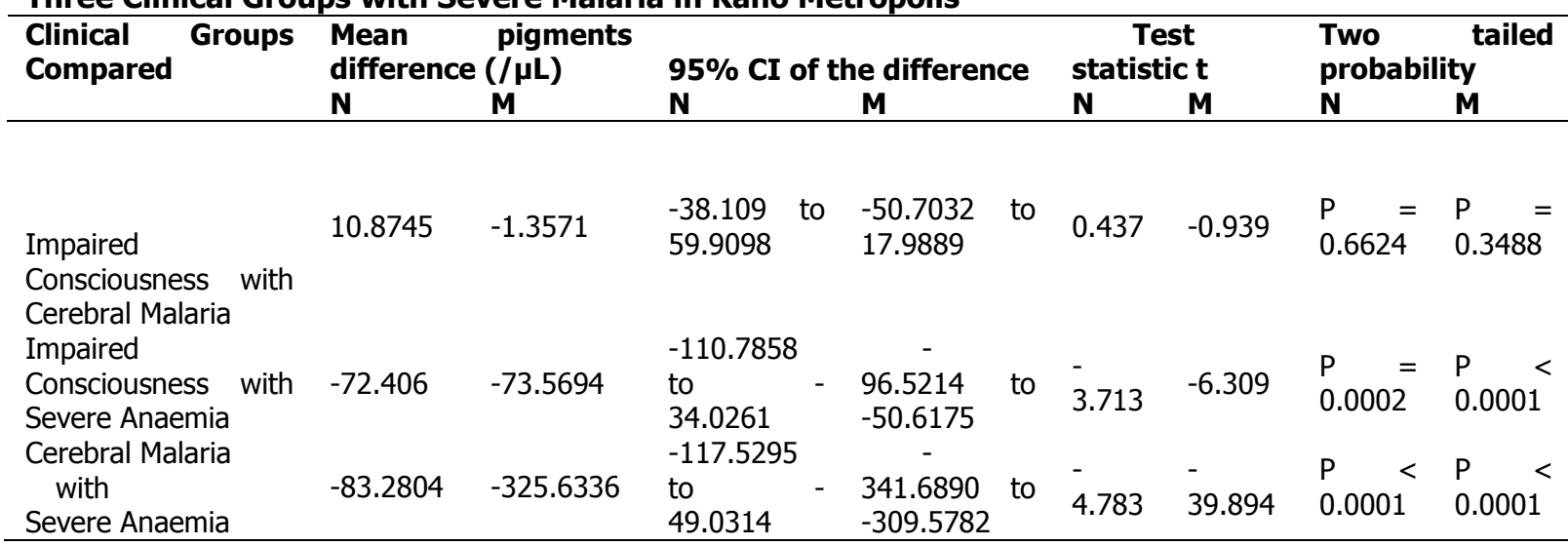

Key: $\mathrm{N}=$ Neutrophilic, $\mathrm{M}=$ Monocytic

Table 4: Comparison of the Mean Haemoglobin Concentration of the Control Group with Three Clinical Groups with Severe Malaria in Kano Metropolis

\begin{tabular}{lcccc}
\hline \multicolumn{4}{c}{ Clinical Groups with Severe Malaria in Kano Metropolis } & $\begin{array}{c}\text { Mean } \\
\text { Haemoglobin } \\
\text { difference } \\
\text { control) }\end{array}$ \\
Clinical Groups & (from & $\mathbf{9 5 \%}$ CI & $\begin{array}{c}\text { Test } \\
\text { statistic t }\end{array}$ & $\begin{array}{c}\text { Level } \\
\text { Significance }\end{array}$ \\
\hline $\begin{array}{l}\text { Impaired } \\
\text { Consciousness }\end{array}$ & -2.8 & -3.19 to -2.41 & -14.027 & $\mathrm{P}<0.001$ \\
Cerebral Malaria & -4.1 & -.42 to -3.78 & -25.456 & $\mathrm{P}<0.001$ \\
Severe Anaemia & -6.8 & -7.01 to 6.59 & -65.018 & $\mathrm{P}<0.0001$ \\
\hline
\end{tabular}


Table 5: Comparison of the Mean Parasitaemia Among Three Clinical Groups with Severe Malaria

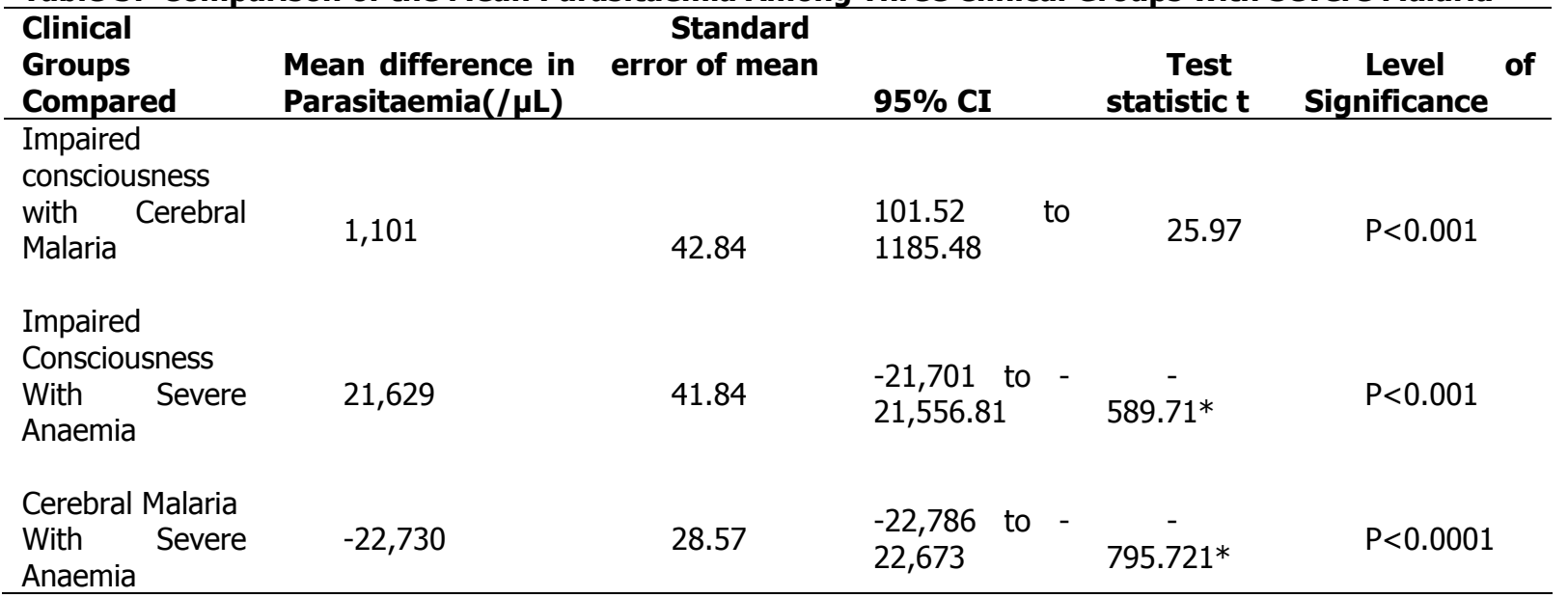

*Statistically Significant

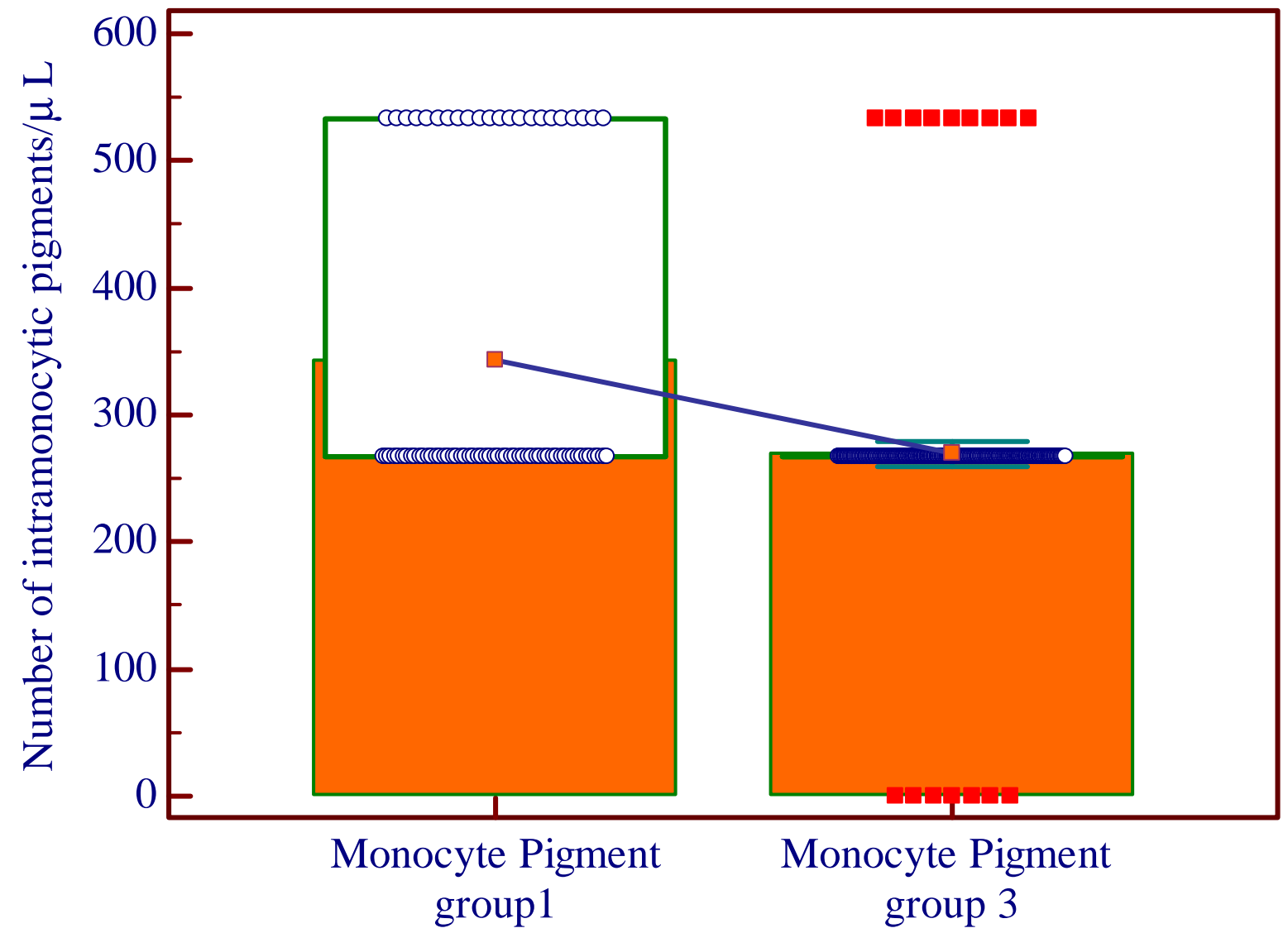

Figure 1: Mann-Whitney's Analysis of Monocytes Pigment Counts Between Impaired Consciousness and Severe Anaemia Groups 


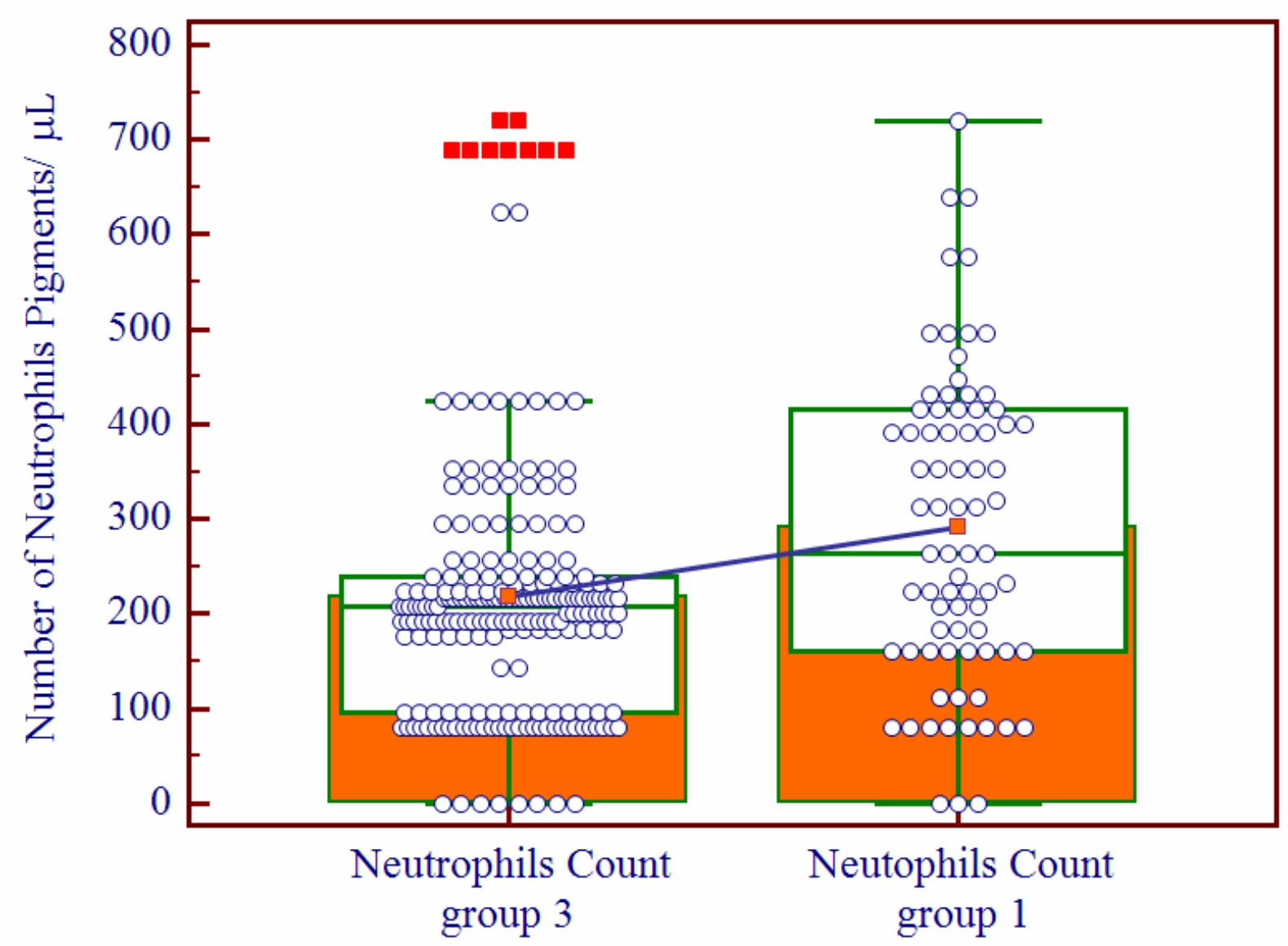

Figure 2: Mann-Whitney's Analysis of Neutrophils Counts Between Impaired Consciousness and Severe Anaemia Groups

\section{DISCUSSION}

Both parasite count and pigment - containing neutrophils counts are useful indicators for severity of malaria infection (Amodu et al., 1998; White et al., 1995; Abubakar et al., 2002). However, the clinical value of parasite count in the diagnosis of individual patients is limited by its poor predictive value (White et al., 1991). This study compared the value of pigment containing leucocytes (neutrophils and monocytes) counts and the malaria parasite density in the prognosis of severe malaria on 420 patients with clinical evidence of severe malaria. Among the three clinical groups studied, patients with impaired consciousness recorded the highest intraleucocytic (monocytic) pigment of $342.86(121.26) / \mu \mathrm{L}$ of blood. Patients with cerebral malaria and other complications $(n=126)$ recorded the highest neutrophils pigment count of $302.10(177.34) / \mu \mathrm{L}$ and the highest parasite count of $234,962(264.5) / \mu \mathrm{L}$. Patients with severe malaria and anaemia had the least pigment count and parasite count of $219.09( \pm 140.96)$ neutrophil pigments/ $\mu \mathrm{L}$ and a parasite count of 212,232 (249.4) $/ \mu \mathrm{L}$ respectively. In all the three groups, a linear relationship between the malaria parasite count and the Intraneutrophilic malaria pigment count in severe malaria was demonstrated.
Slide examination (parasitaemia) has advantage over clinical examination and the data obtained from slides (for parasite density) tend to overestimate the malaria incidence in hyper- endemic area (Goverdhini et al., 1991). Our findings are consistent with the two observations above. It is our belief that the malaria pigment count could serve as a good tool in the diagnosis, management and drug efficacy trials. Microscopy for both intraleucocytic pigments together with parasite count could be valuable in the differential diagnosis of severe malaria in area where uncontrolled use of anti-malaria drugs is widespread (Day et al., 1996).

In a study on 146 Nigerian children with malaria, children with cerebral malaria were found to have the highest median value of $27.0 \%$ of pigment containing monocytes than children with mild (9.0\%), asymptomatic $(6.5 \%)$ or no malaria $(20 . \%)$ (Amodu et al., 1998). The study suggested that pigment containing monocytes and neutrophils count is a simple marker of disease severity in child hood malaria in addition to the parasite count (Amodu et al, 1998). The presence of malaria pigment in monocytes and neutrophils as a marker of disease severity has been validated previously. The association of pigmented neutrophils with cerebral malaria has also been demonstrated (Lyke et al., 2003). 
Our findings of a higher neutrophil pigments count in patients with severe malaria complications and those with cerebral malaria compared to those with severe anaemia corresponds to a previous reports in which peripheral parasitaemia and pigments were found to be strongly correlated with sequestered parasite loads on admission (Ochola et al., 2005). This is in line with our findings. It is evident from our work that both group 1 and 2 had high coma scores (9/15 each) with group 3 having lowest coma score (0/15). At same time group 1 and 2 recorded the higher pigment containing neutrophils count. The difference between group 1 and 2 is insignificant while the two groups differ significantly with group 3 . This work has therefore strongly agreed with this findings (White et al., 1995; White et al., 1991; Abubakar et al., 2002).

Haemolytic anaemia with anisocytosis, poikilocytosis, polychromatia and hyper reticulocytosis has been observed. The anaemia of malaria is most common in children under 5 years of age, older patients with splenomegaly, pregnant women and people with sickle cell disease (Topley, 1998). The products of degranulation and the malaria pigment are toxic to host tissues and may be at least partly responsible for the induction of cerebral malaria (El-Shoura and AlAmari, 1993). Although purified pigment is biologically inert, particulate malaria pigment derived from $P$. falciparum in culture has been shown to release large amounts of tumor necrosis factor (TNF) and interleukin IB (IL IB) (Pichiyangkol et al, 1994). In severe malaria, high plasma concentration of TNF have been shown to correlate strongly with fatal outcome (Kwiatkwoski and Greenwood, 1990). It is likely that some component of malaria parasite membrane, closely adherent to or absorbed on the pigment surface, is a malaria 'toxin' responsible for the cytokine inducing activity (Day et al., 1996).

Particulate pigment could act as a potent vehicle of the delivery of toxin to cytokine producing phagocytes. The cytohedrant 'ghost' and pigment would act as persistent stimulus to local cytokine production in the microvasculature of vital organs (Day et al., 1996). These ultra-structural observations may be relevant, to pathogenesis of coma, which takes place in some of patients with cerebral microvasculature and may explain the discrepancy commonly observed in light microscopy between the amount of apparently extracellular pigment in vessels and that in the peripheral blood (MacPherson et al., 1991). Two types of malaria pigment particles have been demonstrated in intraerythrocytic asexual forms (trophozoites \& schizonts) while a single type was detected in gametocyte ultra-structurally (Pichiyangkol et al., 1994). Catabolism of host haemoglobin by malaria parasite liberates required amino acid

\section{REFERENCES}

Abdool-Gaffar, M. S., Seedat, Y. K., Coovadia, Y. M., Khan, Q. (1992) The White Cell Count in Typhoid fever. Trop Geogr Med. 44: 23-7.

Abubakar, A.G., Kwaru, A. H., Dutse, A. I., Jibril, M. A., Emokpea, M. A. and Takalmawa, H.U. precursors, but it also releases large amounts of potentially toxic haeme that accumulates in parasite food vacuoles during intra erythrocyte development (Eaton and Wood, 1993). The pigment - containing neutrophils count is a simple marker of disease severity in severe malaria in addition to parasite count and does not require special facilities (White et al., 1995; White et al., 1991; Abubakar et al., 2002). It is likely that the pigment containing neutrophil count would remain a relatively robust prognostic indicator in severe malaria at all levels of endemicity whereas monocytes may be less prognostically useful in areas of intense transmission (White et al., 1995). Findings of this study also suggests that the presence of the pigment in monocytes is a valuable marker of recent malaria in patients with negative films for malaria parasites either because of treatment or because the infection is self-resolving. Automated detection of malaria pigment using is therefore a useful malaria diagnostic tool in a semi-rural area. The test could be used in low risk malaria season for diagnosis because of its sensitivity. It can also be used in high risk malaria season for excluding malaria in suspected cases with negative pigment result because of its specificity (De Langen et al., 2006). Although this and previous studies had underscored the significance of malaria pigment count as a reliable indicator of tissue parasite load and disease severity, it should be appreciated that the sensitivity of pigment count may be attenuated by co-morbid tropical infections such as typhoid fever and HIV/AIDS that are associated with neutropenia or pancytopenia, which may result in spuriously low pigment count. Hence, there is the need to interpret pigment count results with caution in such cases (Abdool Gaffar et al., 1992; Bain, 1997).

Findings of this study suggest that pigment containing Leucocytes count could be a good prognostic tool in the measure of malaria disease severity. It would be found useful in clinical trials for the efficacy of antimalarial in addition as an alternative to parasite density estimation in severe malaria.

\section{Recommendations}

Hospitals and health care institutions in malaria endemic zones should endeavor to include pigment detecting haematology auto-analyzer in order to facilitate early identification of potentially severe malaria infection. And the clinicians, whom are traditionally only contented with the parasite count to diagnosis and assess severity of malaria, should be educated on the need to appreciate the significance of the pigment count as a more reliable indicator of disease severity within the context of uncontrolled use of anti-malarial drugs.

(2002) Haematological Markers of Severe P. Falciparum Malaria: An Intraleucocytic Pigment Approach Hamdard Medicus 54(2): 171-75. 
Amodu, O. K., Adeyemo, A.A., Oumese, P. E., Gbadegesin, R. A. (1998): Intraleucocytic Malaria Pigment and Clinical Severity of Malaria in Children. Transaction of the Royal Society of tropical Medicine and Hygiene 92 (1):54-56.

Bain, B. J. (1997). The Haematological Features of HIV Infection. Br J Haematol. 99 :1-8.

Day, N. P., Pham, T. D., Phan, T. L., Dinh, X. S., Pham, P. L., Ly, V. C., Tran, T. H., Nguyen, T. H., Bethell, D. B., Nguyan, H. P., Tran, T. H. and White, N. J. (1996). Clearance Kinetics of Parasites and Pigment-containing Leukocytes in Severe Malaria. Blood 15;88(12):4694-700.

Eaton, J.W. and Wood, P. A. C. (1993). Haemoglobin Catabolism and Host Parasite Heme Balance in Chloroquine-sensitive and Cloroquineresistant Plasmodium berghei Infections . Am J Trop Med Hyg 48:(4) 465-472.

Ejov, M. N., Tun, T., Aung, S., Lwin, S. and Sein, K. (1999). Hospital-based Study of Severe Malaria and Associated Deaths in Myanmar Bulletin of World Health Organisation 77(4) 310-314.

El-shoura, S. M. and Al-Amari O. M. (1993). Falciparum Malaria in Naturally Infected Human Patients: I. Ultrastructural Differences Between Malaria Pigments in Intraerythrocytic Asexual and Sexual Forms. J.Mopho/ 215 (3) $201-206$.

Govardhini, P, Monoharan, A., Subramamian, S., Mohaptra, S. S., Jambulingam, P. and Dos, P. K. (1991). Symptomatic Diagnosis of $P$. falciparum Malaria in Field Conditions. Indian journal of Malariology 28 (1):55-62

Kwiatkowski, D., Hill, A. and Greenwood, B.M. (1990) TNF Concentrations in Fatal Cerebral, Nonfatal Cerebral and Uncomplicated Plasmodium falciparum Malaria. Lancet 336 1201-1204.

Lyke, K. E., Diallo, A., Dicko, A., Coulibaly, D., Guindo, A., Clissoko, Y., Sangare, L., Coulialy, S., Dakuouo, B., Doumbo, O. K. and Plowe, C. V. (2003). Association of Intraleucocytic Plasmodium falciparum Pigment with Disease
Severity, Clinical Manifestation and Prognosis in Severe Malaria Am J Trop Med Hyg. 69 (3) 253-9

MacPherson, G.G., Warrell, M. J. and White, N. J. (1985). Human Cerebral Malaria: A Quantitative Ultra-structural Analysis of Parasitized Erythrocyte Sequestration. $\mathrm{Am} \mathrm{J}$ Pathol 119(3), 385-401.

Ochola. L. B., Marsh, K., Lowe, B., Gal, S., Pluschke, G. and Smith, T. (2005). Estimation of the Sequestered Parasite Load in Severe Malaria Patients Using Both Host and Parasite Markers Parasitology 131(pt4) 449-58

Pichiyangkol, S., Saengkrai, P. and Webster, H. K. (1994). Plasmodium Pigment Induces Monocyte to Release High Levels of Tumor Necrosis Factor and Interleukin -I beta. American Journal of Tropical Medicine and Hygiene 51 94) 430-435.

Topley, E. (1998) Anaemia in Rural Africa: Community Support for Control Activities Where Malaria is Common 12-14 FSG Medic Media, Vine House, Fair Green, Reach Cambridge COJD, UK.

Slater, A. F., Swiggard, W. J., Orton, B. R., Flitter, W. D., Goldberg, D. E., Cerami, A. and Henderson, G. B. (1991). An iron-Carboxylate Bond Links the Haeme Units of Malaria Pigment. Proceedings of the National Academy of Sciences of the U.S.A. 88: 325 329.

White, N. J., Ferguson, D. J. and Nguyen, P. H. (1995). Intraleucocytic Malaria Pigment and Prognosis in Severe malaria. Transaction of the Royal Society of Tropical Medicine and Hygiene 89 (2): 200-204.

White, N. J., Chapman, D. and Watt, G. (1991). The Effects of Multiplication and Synchronicity on the Vascular Distribution of Parasites in falciparum Malaria. Transactions of the Royal Society of Tropical Medicine and Hygiene 86: 590-597.

White, N.J. (1992). Antimalarial Drug Resistance: the Pace Quickens Journal Antimicrobial chemotherapy
30:571-585. 